\title{
Application of Random Vibration Amplitude Vector Method to Damage Identification of Grid Structure
}

\author{
Yalan Huang ${ }^{1, a^{*}}$, Menghong Wang ${ }^{1, b}$, Shiyuan Dong ${ }^{1, c}$ \\ ${ }^{1}$ Department of Civil Engineering, Beijing University of Civil Engineering and Architecture, Beijing \\ 100044, P.R. China \\ ahuangyalan315@126.com, bwangmh@bucea.edu.cn, ${ }^{\text {c8 }}$ w10971669@qq.com
}

Keywords: grid structure; randomly excited; cross correlation function; damage identification Abstract: In this paper, a structural damage identification method, Cross-correlation Function Amplitude Vector (CorV), which based on stochastic process is proposed, it only need the acceleration response signal which obtained from the experimental test of the adjacent measurement points to calculate the cross-correlation between the adjacent measurement amplitude, and combined with certain rules into the $\operatorname{Cor} V$. By defining the degree of change $K$ of $\operatorname{Cor} V$ before and after structural damage, we can judge whether there is damage according to the value. In order to verify the feasibility of the method, a 5-spanning bolt-ball grid structure model was constructed. Using a running trolley to simulate the random excitation, and the truncating of the rod to simulate the complete damage, a single and a multi-point damage under random citation was deployed in the experiment. The experimental result indicates that the proposed method is effective for single and multi-point damage in the grid structure. It is also shown that the method has universal applicability for damage identification of the grid structure under random excitation.

\section{Introduction}

In recent years, space grid structures are widely used in various storage structures of cement and steel industry, as well as super large span structures such as A380 hangar. In addition to the span, these structures sometimes have large numbers of suspension and moving loads. These devices run with constant random excitation of the structure, and the long time random vibration causes the bolt looseness, bar bending, rod fracture and other structural damage. But the grid structure has numerous bars, it is difficult to discover local damage by eyes, and the global collapse caused by accumulation of local damage happens from time to time. It is available to use dynamic testing to predict the location of the damage for the space structures[1]. In this paper, the method combining the theory of random vibration with damage identification was applied, and model experiment was perfomed, which verified the applicability of the method.

In this paper, the basic principle of amplitude vector based on cross correlation function has been applied for identification of damage of space truss structure. This method only need to obtain the accerleration response signal of the adjacent measuring points on the basis of the experimental test, then calculate the cross correlation function amplitude between adjacent points and construct the cross correlation function amplitude vector $(\mathrm{CorV})$ by certain rules. The amplitudes of $\operatorname{CorV}$ related to the location of measuring points can be regarded as the generalized modal of the structure, which reflect the spatial distribution of structural vibration information[2]. Therefore, the nature and characteristics of $\operatorname{Cor} V$ can be used to identify structural damage.

\section{The principle of cross correlation function amplitude vector}

Definition of cross correlation function. Under the random vibration action of the structure, if the acceleration response of $N$ measuring points can be measured at the same time, and the acceleration response of any two measuring points can be regarded as real stationary stochastic processes like $x_{1}(t)$ and $x_{2}(t)$. If their joint distribution probability is $p\left(x_{1}, x_{2}\right)$, then the cross-correlation functions of two different stochastic processes are:

$$
\mathrm{R}_{\mathrm{x}_{1} \mathrm{x}_{2}}(\tau)=\mathrm{E}\left[\mathrm{x}_{1}(\mathrm{t}) \mathrm{x}_{2}(\mathrm{t}+\tau)\right]=\int_{-\infty}^{+\infty} \int_{-\infty}^{+\infty} \mathrm{x}_{1} \mathrm{x}_{2} \mathrm{p}\left(\mathrm{x}_{1}, \mathrm{x}_{2}\right) d \mathrm{x}_{1} \mathrm{dx} \mathrm{x}_{2}
$$


If $x_{1}(t)$ and $x_{2}(t)$ satisfy the ergodic hypothesis, , $\hat{x}_{1}(t)$ and $\hat{x}_{2}(t)$ are the two samples, then (1) can be expressed as[3]:

$$
\mathrm{R}_{\mathrm{x}_{1} \mathrm{x}_{2}}(\tau)=\lim _{\mathrm{T} \rightarrow \infty} \frac{1}{\mathrm{~T}} \int_{0}^{\mathrm{T}} \hat{\mathrm{x}}_{1}(\mathrm{t}) \hat{\mathrm{x}}_{2}(\mathrm{t}+\tau) \mathrm{dt}
$$

It is assuming that $x_{1}(t)$ and $x_{2}(t)$ are two different measured random processes and $T$ is the sampling time, then dispersing $x_{1}(t)$ and $x_{2}(t)$ with equal time interval $\Delta t$. It will obtain $N$ dispersed sampling values $x_{1}\left(t_{n}\right)$ and $x_{2}\left(t_{n}\right)$, and the number of sample points is $N=\frac{T}{\Delta t}+1$, the cross-correlation function can be expressed as:

$$
\mathrm{R}_{\mathrm{x}_{1} \mathrm{x}_{2}}(\mathrm{k})=\frac{1}{\mathrm{~N}} \sum_{\mathrm{i}-1}^{\mathrm{N}-\mathrm{k}} \mathrm{x}_{1}(\mathrm{i}) \mathrm{x}_{2}(\mathrm{i}+\mathrm{k}) \quad(\mathrm{k}=0,1,2, \ldots, \mathrm{N})
$$

The basic principle of amplitude vector of cross-correlation function. Lei Jiayan of the Beijing Jiaotong University presented in the literature[4] to calculate the cross correlation function amplitude between the dynamic responses of any two adjacent points:

$$
\mathrm{r}_{\mathrm{i}, \mathrm{i}+1}=\left|\mathrm{R}_{\mathrm{x}_{\mathrm{i}} \mathrm{x}_{\mathrm{i}+1}}(\mathrm{t})\right|_{\max }=\left|\mathrm{R}_{\mathrm{x}_{\mathrm{i}} \mathrm{x}_{\mathrm{i}+1}}(\mathrm{~m})\right|_{\max }
$$

If $n$ measurement points are arranged on the structure, the acceleration response data of each measurement point are obtained, and the cross-correlation function amplitude between the adjacent measurement points is calculated, and the amplitude of $n-1$ cross-correlation function can be obtained. A certain rule of the composition of the cross-correlation function amplitude vector, denoted as $\operatorname{Cor} V$, is:

$$
\operatorname{CorV}=\left[r_{1,2}, r_{2,3}, \ldots, r_{n-1, n}\right]
$$

Judgment basis of damage identification. When the spectral properties of external excitations are constant, each vector element $r_{i, i+1}$ has a fixed proportion relation in CorV. CorV graphs have similar shapes under different damage states varying degrees of change only at the damage location, which menas $\operatorname{CorV}$ has a fixed morphology[5].

In order to make the damage identification more convenient and accurate, the cross correlation function amplitude vector $\operatorname{CorV}$ is normalized before and after structural damage, which makes it more omparable, then:

$$
\operatorname{CorV}_{*}=\frac{\operatorname{CorV}(\mathrm{i})}{\left[\sum \operatorname{CorV}^{2}(\mathrm{i})\right]^{\frac{1}{2}}}
$$

To locate the damaged point, the changing degree of $\operatorname{CorV}_{u}$ and $\operatorname{CorV}_{d}$ before and after damage is recorded as $K[6]$ :

$$
\mathrm{K}(\mathrm{i})=\left|\frac{\operatorname{CorV}_{\mathrm{d}}(\mathrm{i})-\operatorname{CorV}_{\mathrm{u}}(\mathrm{i})}{\operatorname{CorV}_{\mathrm{u}}(\mathrm{i})} \times 100 \%\right|
$$

$\operatorname{CorV}_{u}(i)$ and $\operatorname{CorV}_{d}(i)$ respectively represent the vector factor in the amplitude vector of cross correlation function of damaged and undamaged structure in the formula. As a damage index, $K$ has a one-to-one correspondence relationship with the testing rod. Where there is a maximum value of $K$, there is possibly a damaging point, which can be used as a basis for accurating the damage.

\section{Experimental analysis}

The general situation of model and instrument. In this paper, a grid structure model is used to study the damage identification. The model is summarized as follows: The grid structure size of $3 \mathrm{~m}$ $\times 5 \mathrm{~m}$, it havs 60 points and 174 members, All section dimensions are $\Phi 42 \mathrm{~mm} \times 2.5 \mathrm{~mm}$, only the length is different, the steel used is Q235, and the elastic modulus is E=200GPa. All rods and joints in the model are connected in a hinged manner and the four supports are fixed. The grid structure along the longitudinal chord node installed two track channel, and welded angle car, which can make it move back and forth on the track, and exert an incentive effect on the structure(As shown in fig. land fig. 2). 


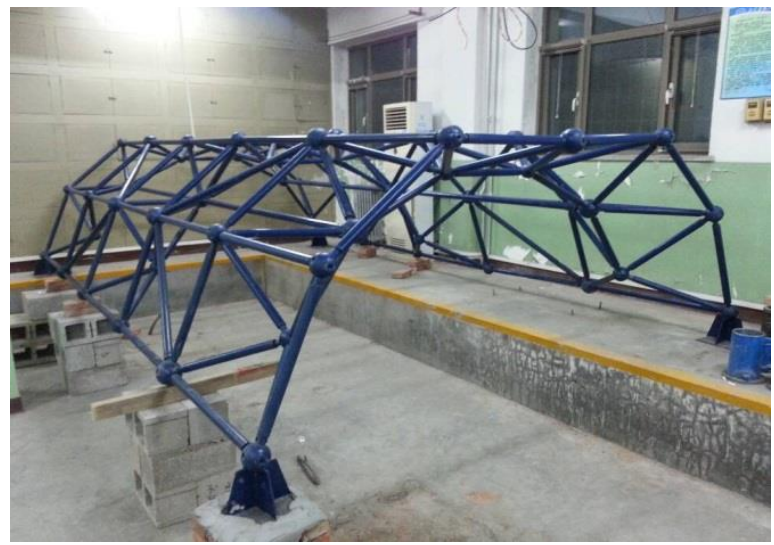

Fig. 1 Truss structure model

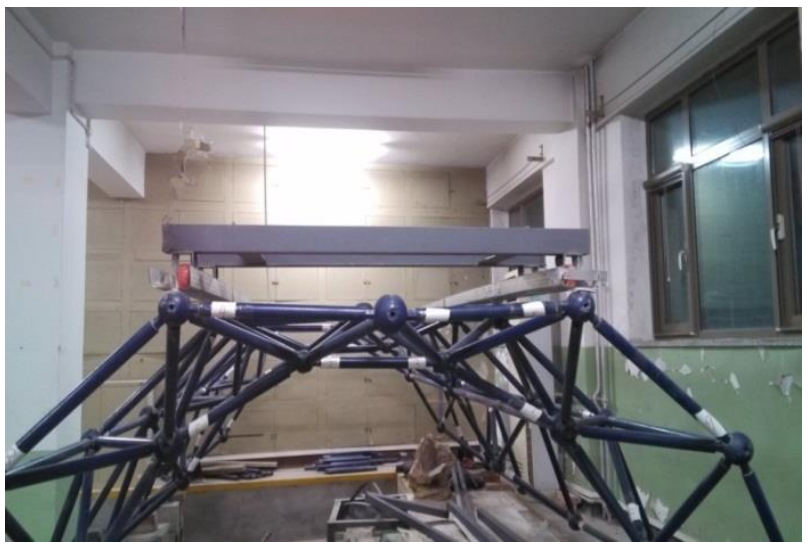

Fig. 2 Excitation device

Selection of adjacent measuring points. In this paper, the abdominal bar is used as an example, and the nodes are marked as shown in Fig. 3. The nodes serial numbers are 19, 10, 17, 6, 15, 2, 14, $3,16,7,18,11,20$, and between the nodes are abdominal bars. The rods are marked as shown in Fig. 4. The adjacent nodes are selected according to the node sorting, and the cross-correlation function amplitudes between adjacent nodes are calculated.

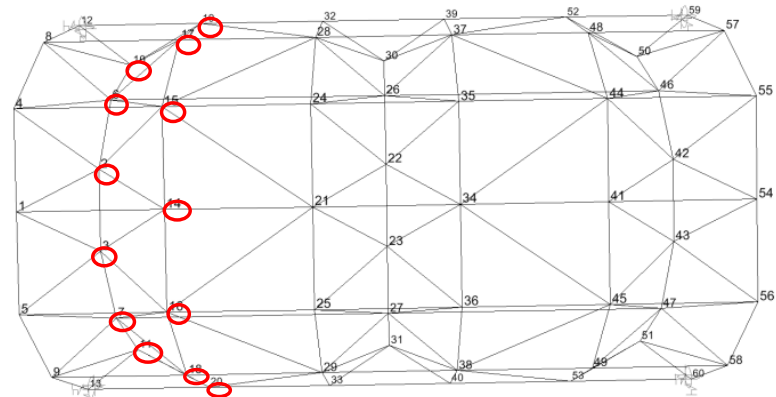

Fig. 3 Node numbering in grid structure

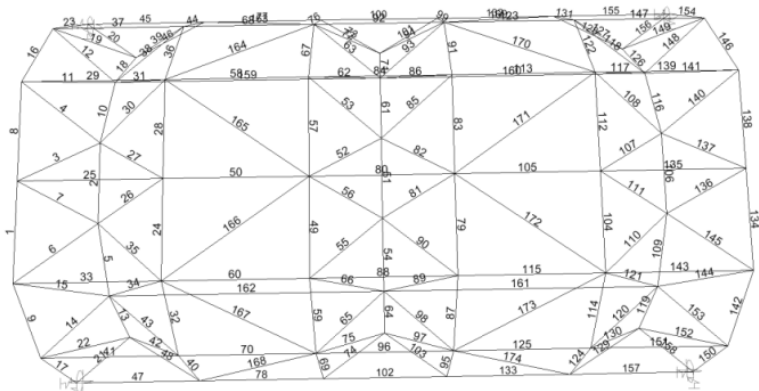

Fig. 4 Member numbering in grid structure

\section{Damage identification}

(1) Damage condition

In this paper, complete fracture is taken as structural damage, and two damage cases are set up:

Condition 1: Damage of No. 34 pole (abdominal bar), the number of nodes at both ends are 7 and 16. The node number in the detection area of the damaged member is sorted as: 19, 10, 17, 6 , $15,2,14,3,16,7,18,11,20$. The inter node number is sorted as: 46, 39, 38, 31, 30, 27, 26, 35, 34, 43, 42, 48. There are 13 nodes and 12 member bars.

Condition 2: Simultaneous damage on rod 34 and rod 35 (abdominal bar), rods 34 and 35 have common joints. The numbers of nodes connected at both ends are 7 and 16, 3 and 16 respectively, The node number of the detection area in which the damage member is located is the same as that of the case number sorting and the working condition.

(2) Damage identification

First of all, the cross correlation function amplitudes before and after structural damage can be calculated by formula (3) respectively. After normalization, the cross correlation function amplitude $r_{i, i+1}$ of the undamaged and damaged structure in the same area can be obtained. In the order of node number, $\operatorname{CorV}_{k}$ can be formed by $r_{i, i+1}$. After obtaining the $\operatorname{CorV}_{d}$ and $\operatorname{CorV}_{u}$ of damaged and undamaged structure, we substitute $\operatorname{CorV}_{d}(i)$ and $\operatorname{CorV}_{u}(i)$ into formula (7), and calculate the relative change value $K$ of damaged and undamaged situations, as shown in Tab. 1 and Tab. 2: 
Tab.1 Change value $K$ of $\operatorname{Cor} V$ in condition 1

\begin{tabular}{c|c|c|c|c|c|c}
\hline node & $19-10$ & $10-17$ & $17-6$ & $6-15$ & $15-2$ & $2-14$ \\
\hline bar & 46 & 39 & 38 & 31 & 30 & 27 \\
\hline $\mathrm{K}$ & 0.0914 & 1.1954 & 0.2294 & 0.3834 & 0.3728 & 0.0244 \\
\hline \hline node & $14-3$ & $3-16$ & $16-7$ & $7-18$ & $18-11$ & $11-20$ \\
\hline bar & 26 & 35 & 34 & 43 & 42 & 48 \\
\hline $\mathrm{K}$ & 0.6785 & 1.2196 & 2.3691 & 1.4533 & 0.9906 & 0.2648 \\
\hline
\end{tabular}

Tab.1 Change value $K$ of $\operatorname{Cor} V$ in condition 2

\begin{tabular}{c|c|c|c|c|c|c}
\hline node & $19-10$ & $10-17$ & $17-6$ & $6-15$ & $15-2$ & $2-14$ \\
\hline bar & 46 & 39 & 38 & 31 & 30 & 27 \\
\hline $\mathrm{K}$ & 0.6888 & 0.9215 & 0.5269 & 0.2523 & 1.6873 & 5.0476 \\
\hline \hline node & $14-3$ & $3-16$ & $16-7$ & $7-18$ & $18-11$ & $11-20$ \\
\hline bar & 26 & 35 & 34 & 43 & 42 & 48 \\
\hline $\mathrm{K}$ & 5.6311 & 10.822 & 11.331 & 2.7236 & 0.6291 & 0.1952 \\
\hline
\end{tabular}

According to the data in the table, it is impossible to see the specific location of the damage of the structure. But using the relative change value $K$ under different working conditions as a line chart, can obtain the damage location of the structure more intuitively. The line graph of relative change value $K$ under different working conditions is shown in Fig. 6 and Fig. 7.

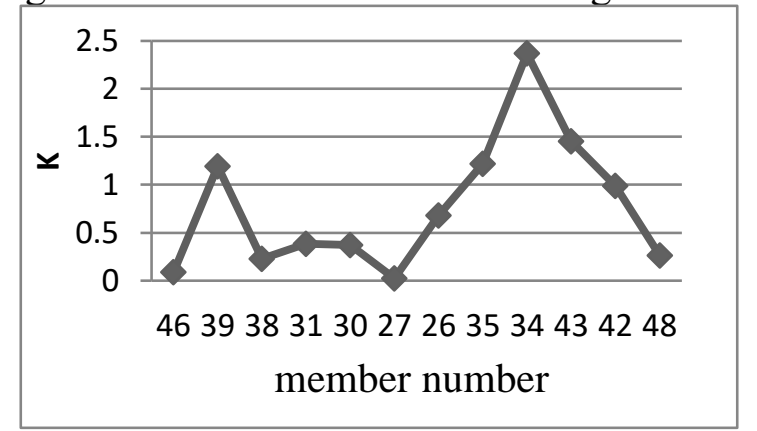

Fig. 6 Curve of $K$ in condition 1

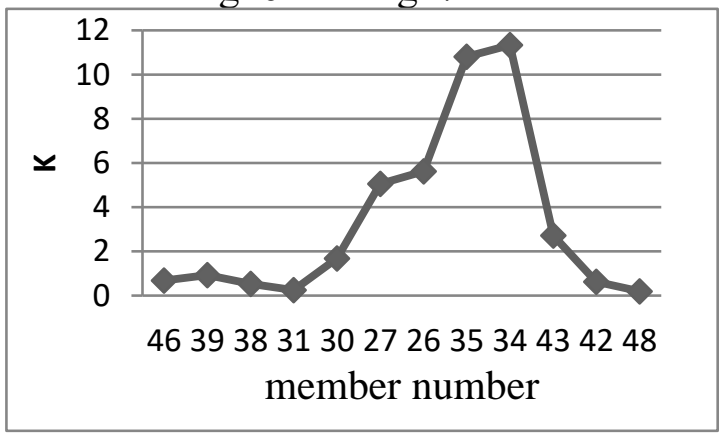

Fig. 7 Curve of $K$ in condition 2

As can be seen from Fig. 6, the peak value of the $K$ appears at rod 34. As can be seen in Fig. 7 , the peak value of the $K$ appears at the bar 34 and 35 also. According to the foregoing principle, The point at which $K$ peaks is the damage to the structure. It is consistent with the setting of the damage condition, which means that the damage detection method of space truss structure based on the principle of cross correlation function amplitude vector is proved to be feasible.

\section{Conclusion}

This paper draws the following conclusions:

(1) It is proved that the damage identification method based on the random vibration amplitude vector is feasible for the damage identification of the grid structure under random excitation of the equipment;

(2) The random excitation of equipment and the moving load applied to the structure during actual situation is simulated by the running of the trolley on the grid structure, which provides a reference for the damage identification of the active grid structure;

(3) By selecting the adjacent measurement points In the calculation of cross-correlation function amplitude, a one-to-one relationship between the calculated amplitude and the rod between measured node was established. In the identification process, the damage location can directly determined according to the maximum value of $\mathrm{K}$, which made the process simple and effective. 


\section{Acknowledgements}

This research was financially sponsored by National Natural Science Foundation (51578038), study on Damage Identification Lose Efficacy Mechanism and Repair Strategy of Space Truss Structures under Facility Excitations.

\section{References}

[1] SUN X D, OU J P, Assessment of vibration-based da-mage indexes in structural health monitoring, J. Journal of Vibration and Shock. 28 (2000) 9-13.

[2] YANG Z C, DANG X J, WANG L, et al, Experi-mental investigation on a combined damage localization method for composite beams and plates, J. Journal of Vib-ration, Measurement \& Diagnosis. 30 (2010) 115-118.

[3] ZHU W Q, Random Vibration, Science Press, Beijing, 1998.

[4] Lei J Y, Structural damage detection from cross-correlation function analysis of stochastic responses, Bei-jing Jiaotong University, Beijing, 2011.

[5] YU Z F, YANG Z C, Correlation function amplitude vector and its application to structural damage detection, J. Mechanical Science and Technology for Aerospace E-ngineering. 25 (2006) 24-27.

[6] HOU Y F, HU W B, Damage detection of historic ti-mber structure based on cross correlation function amplit-ude vectors, Review of the Chinese conference on t-heoretical and applied mechanics-2001 In memorial of TS-IEN HSUE-SHEN's 100th anniversary, 2011. 